\title{
Conditions d'émergence et de pérennisation des services d'appui à la traction animale en Afrique subsaharienne : cas des agroéquipements
}

\author{
R. Pirot ${ }^{1}$ M. Havard ${ }^{1}$ E. Vall ${ }^{1}$ \\ G.A. Kemtsop ${ }^{2}$ A. Fall ${ }^{3}$
}

Mots-clés

Energie animale - Matériel agricole - Entretien - Réparation Atelier - Petite entreprise Marché - Burkina Faso Cameroun - Sénégal.

\begin{abstract}
Résumé
A partir des années 1960, au Burkina Faso, au Nord-Cameroun et au Sénégal, des programmes d'équipement des paysans en matériels de traction animale, alimentés par l'importation, puis par les fabrications industrielles locales, ont été financés par les cultures d'arachide et de coton. Dans les années 1980 et 1990, la réduction drastique des financements de ces programmes, particulièrement au Burkina Faso et au Sénégal, a mis en difficulté les industries nationales, contraintes de restructurer et diversifier leur outil de production. La situation s'est aggravée avec la dévaluation du franc Cfa qui a entraîné une augmentation des prix des matériels. Face à ces évolutions brutales, les artisans du fer ont réagi en prenant en charge progressivement l'entretien et les réparations des matériels agricoles de traction animale. Puis ils se sont mis à produire avec des matériaux de récupération des agroéquipements à des prix compatibles avec les moyens financiers des agriculteurs. Cependant la pérennisation du secteur artisanal des agroéquipements ne pourra être assurée que par l'amélioration de la qualité des produits, de la gestion et des performances des ateliers, ainsi que par la formation de groupements d'artisans pour faciliter I'approvisionnement en matière première et I'accès aux marchés. En parallèle, les organisations paysannes doivent chercher à structurer et formaliser la demande des paysans en agroéquipements.
\end{abstract}

\section{INTRODUCTION}

A l'aube du $\mathrm{XXI}^{\mathrm{e}}$ siècle, la traction animale continue sa progression en Afrique de l'Ouest et centrale. Cette technologie répond aux besoins de la majorité des exploitations agricoles familiales de polyculture élevage, dont les superficies cultivées sont généralement comprises entre deux et quinze hectares. La traction animale est répandue dans les zones sahéliennes et de savanes. Ainsi, au Sénégal, au Mali, au Burkina Faso et en zone cotonnière du Cameroun, il y a un attelage pour moins de dix hectares cultivés.

Pour accompagner la diffusion des matériels agricoles, les programmes mis en place par les structures des Etats s'appuyaient sur des cultures commerciales (arachide au Sénégal, coton au Burkina Faso et au Cameroun) et intégraient les services d'appui nécessaires

1. Cirad, avenue d'Agropolis, 34398 Montpellier Cedex 5, France

2. Sadel-GIE, BP 293, Garoua, Cameroun

3. Isra, BP 240, Saint-Louis, Sénégal (fabrication, distribution et financement des matériels agricoles). Mais avec la mise en œuvre des politiques de désengagement de l'Etat dans les années 1980, ces services ont été repris par des privés, totalement au Sénégal et partiellement au Burkina Faso, alors qu'au Nord-Cameroun, la Société de développement du coton (Sodécoton) continue à les assurer. Quelles sont alors les répercussions de ces changements sur l'émergence et la pérennisation de services d'appui à la traction animale ?

Cet article présente les résultats des études (bibliographie, enquêtes et suivis) sur l'artisanat du fer réalisées au Cameroun $(8,11,14)$ et au Sénégal (2) dans le cadre d'une action thématique programmée (ATP) du Cirad réalisée de 1999 à 2001, et ceux d'une étude sur la mécanisation menée par la FAO au Burkina en 1997 (1). Dans chaque pays, une mise en perspective historique de l'approvisionnement des paysans en matériels agricoles de traction animale a été préalablement réalisée. Les enquêtes, entretiens et suivis avec les artisans ont fait l'objet d'analyses spécifiques afin de comparer les différents types d'artisans selon leur implication dans l'entretien et la fabrication des matériels de traction animale. 
- IMPACT DES POLITIQUES DES ETATS SUR LE SECTEUR DES AGROEQUIPEMENTS DE TRACTION ANIMALE

Les politiques de désengagement des Etats du secteur des agroéquipements ont favorisé le développement de l'artisanat du fer. Le cœur du marché des agroéquipements s'est déplacé du groupe groupements/sociétés de développement/fournisseurs industriels et importateurs vers le groupe paysans/artisans selon des trajectoires spécifiques à chaque pays.

\section{Rôle de la Sodécoton au Nord-Cameroun}

La Compagnie française de développement de la fibre textile (Cfdt), de 1950 à 1973, puis la Sodécoton, à partir de 1974, ont fortement contribué à la promotion de la traction animale grâce à un encadrement soutenu des paysans et aux crédits d'équipement octroyés aux paysans $(8,13)$. A partir de 1985 , la traction animale se diversifie avec l'utilisation de la traction asine. En 1989, la crise mondiale du coton a entraîné la baisse du prix d'achat du coton au paysan. En 1994, la dévaluation du franc Cfa a remonté de 59 p. 100 le prix d'achat du coton aux paysans mais a fait doubler les prix des charrues et des intrants agricoles. Dans ce contexte défavorable, la Sodécoton a opté pour le maintien des crédits à l'équipement pour accompagner la progression de la traction animale. Aujourd'hui, le recours à la location d'attelage permet à la majorité des paysans d'avoir recours à la traction animale, alors que seulement un quart des exploitations agricoles sont équipées.

La Sodécoton a toujours passé les commandes agroéquipements aux industriels. Ce fut d'abord à la Société camerounaise de métallurgie $(\mathrm{Scdm})$ qui n'effectuait que le montage des charrues, des charrettes, des ensembles sarcleurs et des butteurs. En 1994, le relais a été pris par une industrie basée au Nord-Cameroun, Manucycle (fabricant de bicyclettes, de pousse-pousse et de moulins).

Les premiers forgerons de la région fabriquaient des armes de guerre, certains ustensiles ménagers, les outils agricoles manuels et quelques pièces d'usure des matériels agricoles de traction animale. A partir de 1980-81, la Sodécoton a cherché à appuyer les artisans forgerons en formant des encadreurs chargés de leur apprendre la fabrication des pièces d'usure et la réparation des matériels. Elle leur fournissait le bois et le fer. En 1985, ce projet s'est arrêté faute de financement. Bien qu'ils aient reçu peu d'appuis, les artisans forgerons assurent aujourd'hui l'entretien de la majorité des matériels (fabrication des pièces et réparations).

\section{Evolution du secteur des agroéquipements au Sénégal De 1960 à 1980}

L'Etat met en place et contrôle les structures nécessaires au développement de la traction animale autour de la culture arachidière (figure 1A). Au Centre de recherches agronomiques de Bambey, les premiers essais de traction asine, de houes et de semoirs sont réalisés dès 1922. Le semoir rencontre immédiatement un grand succès car il facilite les semis. La charrette devient le moyen de transport privilégié des produits. Avant 1963, tous les équipements agricoles étaient importés de France et les artisans intervenaient très peu dans leur entretien et leur réparation.

Avec l'indépendance, s'installe le programme agricole (PA) qui a pour objectif d'accélérer l'équipement du paysannat en matériels et intrants. Dès 1960, se met en place l'Office de commercialisation agricole (OCA), qui s'appuie sur les coopératives avec caution solidaire des adhérents, pour développer sa politique de crédit, de subventions et de commercialisation. Il cède sa place à l'Office national de la commercialisation et de l'assistance au développement (Oncad) en 1966. En 1963, l'Etat crée la Société industrielle sénégalaise de constructions mécaniques et de matériels agricoles (Siscoma) qui bénéficie d'un quasi-monopole compte tenu de la procédure mise en place pour les commandes de matériels.

Dans les années 1960-70, les actions en faveur des artisans forgerons ont été essentiellement des formations pour l'entretien des matériels agricoles de traction animale. Elles sont à mettre à l'actif de la Siscoma, du Bureau international du travail (BIT), de la direction et des services de la formation professionnelle rurale. Cette profession s'est bien développée dans les milieux urbains, où les ateliers du fer, couramment appelés «menuiserie métallique », ont du travail assuré toute l'année et l'approvisionnement en matière première est assez facile. Après les formations, les forgerons villageois qui retournent à leur forge ne peuvent appliquer les techniques apprises faute d'équipements adaptés. Beaucoup s'installent alors en zone urbaine dans les menuiseries métalliques $(6,10)$. Dans les années 1970 face à ce constat, des actions « forgerons villageois » ont été prises en charge par la Société de développement et de vulgarisation agricole (Sodeva), la Sodefitex, et le Projet riz Sédhiou (PRS). L'intervention porte sur la formation, l'approvisionnement en matière première, la fourniture de moyens de fonctionnement et l'aide à l'équipement par l'octroi d'un crédit. Cette approche nécessitant un encadrement rapproché et des moyens importants n'a touché que des échantillons limités et n'a pu être étendue faute de moyens (5). En vingt ans, plus de 900000 unités comprenant des semoirs, des houes, des souleveuses et des charrettes ont été diffusées.

\section{Depuis 1980}

A partir de 1980 s'est opéré le démantèlement du secteur des agroéquipements, excepté pour l'artisanat du fer (figure 1B). En 1980, la dette des coopératives arachidières à l'Oncad s'élève à 32 milliards de francs Cfa, dont 40 p. 100 pour les équipements de traction animale, avec, pour conséquence, l'arrêt du PA qui provoque le démantèlement du système d'approvisionnement et de fabrication des matériels agricoles: l'Oncad est dissout et la Siscoma dépose son bilan. Les artisans deviennent les seuls interlocuteurs des paysans pour l'entretien des équipements agricoles.

En 1985, la Nouvelle politique agricole, visant la libéralisation $\mathrm{du}$ secteur arachidier, est mise en place. Les producteurs doivent faire leur demande de crédit directement à la Caisse nationale de crédit agricole du Sénégal (Cncas). Face à la chute de la production d'arachide, l'Etat propose un plan de relance de l'arachide dans son programme d'ajustement sectoriel de l'agriculture. Mais ce plan intervient dans un climat d'incertitude peu favorable aux investissements : politique erratique des prix, baisse de fertilité des sols, baisse de qualité des semences, usure des matériels agricoles. Il en résulte que ces vingt dernières années, moins de 100000 unités de matériels agricoles ont effectivement été financées. Malgré cette baisse importante de diffusion des matériels, le taux d'équipement moyen par exploitation n'a pas bougé dans le bassin arachidier grâce au développement important de l'artisanat du fer $(4,12)$. En effet, les artisans assurent non sans difficultés, d'une part, la fourniture régulière de matériels et, d'autre part, l'entretien et les réparations de plusieurs centaines de milliers d'équipements agricoles dans le bassin arachidier ; toutefois l'état général du parc se détériore (7).

\section{Forte implication de l'Etat dans le secteur des agroéquipements au Burkina Faso}

Depuis l'indépendance, les politiques de développement agricole ont visé l'accroissement de la production. En 1965, une option avait été prise en faveur de la traction animale à travers la création des organismes régionaux de développement (ORD). 
Situation A : forte implication de l'Etat dans le marché des agroéquipements

\begin{tabular}{|c|c|c|c|}
\hline $\begin{array}{l}\text { Regroupement } \\
\text { des commandes }\end{array}$ & Marché structuré et centralise & & $\begin{array}{l}\text { Offre importante } \\
\text { et concentrée }\end{array}$ \\
\hline \multirow[b]{2}{*}{$\begin{array}{l}\text { Demande Eq } \\
\text { crédit }\end{array}$} & $\begin{array}{l}\text { Banques, projets } \\
\qquad \text { Offre crédit }\end{array}$ & & \multirow{3}{*}{ Eq } \\
\hline & Sociétés de & Offre Eq & \\
\hline $\begin{array}{l}\text { Groupements } \\
\text { Associations } \\
\text { Coopératives }\end{array}$ & \multirow{2}{*}{$\begin{array}{l}\text { développement agricole } \\
\text { Elaboration demande, } \\
\text { commandes, financement } \\
\text { et distribution des matériels. } \\
\text { Commercialisation } \\
\text { des produits agricoles }\end{array}$} & \multirow[b]{2}{*}{ Offre Eq } & \\
\hline Demande Eq & & & $\begin{array}{l}\text { Industries } \\
\text { nationales }\end{array}$ \\
\hline Paysans $<$ & le proximité très limité pour les $n$ & $\begin{array}{l}\text { matériels, } \\
\cdots \text { répa........ } \\
\text { reations } \\
\end{array}$ & $\Rightarrow$ Artisans \\
\hline
\end{tabular}

$$
\text { B Rupture, déséquilibre }
$$

Situation B : processus de privatisation du marché des agroéquipements

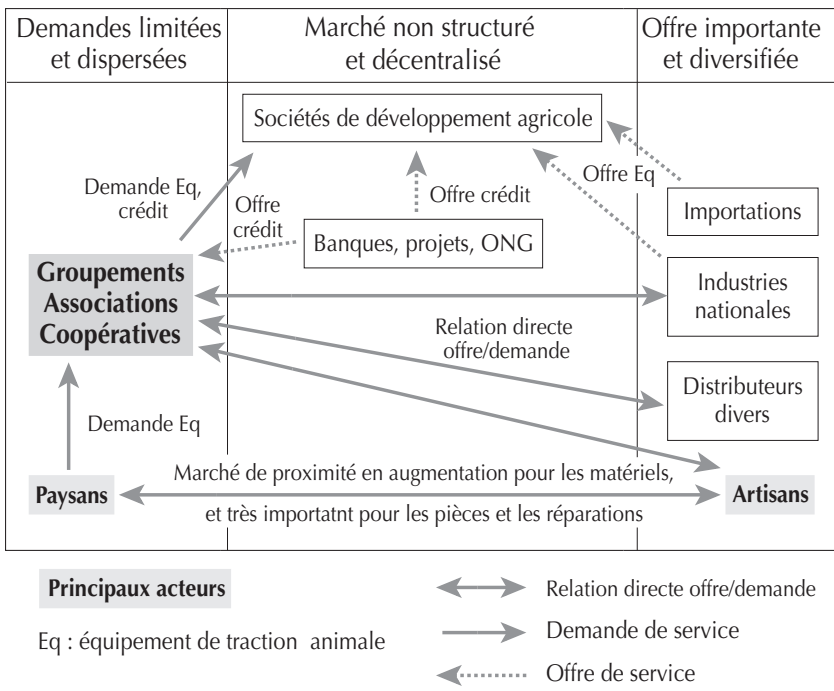

Figure 1 : évolution du secteur des agroéquipements avec le désengagement des Etats.

\section{Appui au financement de l'équipement des paysans}

La politique agricole visait la réalisation de l'autosuffisance alimentaire. Elle prônait la promotion de la traction animale dans le cadre d'une intégration agriculture élevage.

A partir de 1975, avec l'aide d'institutions internationales [Conseil de l'entente, Caisse nationale de crédit agricole (Cnca), coopération suisse...], le rôle de l'Etat a été déterminant dans le financement de l'équipement des producteurs. En 1980, les effectifs d'animaux de trait étaient estimés à 140000 têtes et ceux des matériels agricoles à 135000 unités.

Entre 1980 et 1987, la Cnca a octroyé 3,4 milliards de Fcfa pour la traction animale, dont la majorité sur la zone cotonnière. Elle octroyait les crédits aux ORD et commandait le matériel agricole aux fournisseurs qui livraient directement aux groupements villageois. Le paysan individuel n'avait pas la possibilité de s'adresser à un autre fournisseur. Manquant de fonds pour s'approvisionner au comptant sur le marché, le paysan n'avait que la solution du crédit qui lui permettait d'étaler le paiement sur plusieurs années. Ainsi certains paysans formulaient des demandes, sans avoir réellement besoin des matériels qu'ils revendaient à la première occasion. Cette organisation a été arrêtée à cause de médiocres résultats financiers, mais elle s'est traduite par une augmentation importante du nombre d'attelages. En 1990, les effectifs d'animaux de trait et de matériels agricoles étaient estimés respectivement à 440000 têtes et 425000 unités.

La dévaluation de 1994 a fait doubler les prix des matières premières et des matériels agricoles, alors que le prix du coton n'a augmenté que de 15 p. 100 et celui des céréales n'a pas bougé. Ceci a eu pour conséquence une réduction des demandes d'équipement des paysans. Malgré la Lettre de politique de développement agricole (Lpda) disant que l'Etat doit laisser au secteur privé le soin de fabriquer, importer et distribuer le matériel agricole, l'Etat a continué à jouer un rôle important. Après la dévaluation, il met en place l'opération « 2000 multiculteurs » pour continuer celle des « 30000 charrues » financée par l'Autriche. En 1997, les effectifs d'animaux de trait et de matériels agricoles étaient estimés respectivement à 650000 têtes et à 630000 unités.

\section{Une réelle politique de promotion de l'artisanat}

L'Etat a joué un rôle majeur dans la fabrication et l'entretien des agroéquipements : importation de matière d'œuvre pour les entreprises publiques, fabrication des matériels, formation des artisans. Ainsi, le ministère du Commerce, de l'Industrie et de l'Artisanat, par le biais de la direction générale des Artisans et PME, assure la coordination de la Commission nationale de promotion de l'artisanat (Cnpa) qui, en 1996, a remplacé la Commission interministérielle sur la politique de promotion de l'artisanat (Cippa).

A ses débuts en 1971, le Centre national de perfectionnement des artisans ruraux (Cnpar) était constitué de sections de formation en menuiserie, maçonnerie, forge et mécanique, mais aussi d'un service d'assistance, conseil et soutien aux artisans pour les approvisionner en outils et pièces détachées, et de l'Atelier pilote de construction de matériel agricole (Apicoma). Entre 1978 et 1993, le Cnpar a équipé à crédit plus de 600 artisans formés à la fabrication et aux réparations des matériels de traction animale. Cependant, les crédits individuels d'équipement ont été suspendus en 1988 à cause de difficultés de recouvrement. Jusqu'en 1989, les prix de vente des magasins d'outillage et de matière première du Cnpar étaient inférieurs à ceux du marché, car ils s'approvisionnaient en hors taxe.

Depuis les années 1990, les programmes en faveur des PME et des artisans regroupent plusieurs aspects : formation (forge, mécanique tracteurs, soudure, fonderie, alphabétisation, gestion), équipement à crédit et, depuis peu, grâce à des fonds de roulement pour l'achat de matière première, accès à des marchés des projets et des $\mathrm{ONG}$, et appui à l'organisation de la profession. Les différents programmes n'ont pas la même approche. Le Projet de promotion des entreprises rurales de construction métallique et des mécaniciens (Percomm), très proche des artisans, privilégie une approche individualiste avant d'aller vers le groupement. Le PAB (Programme de promotion de l'artisanat au Burkina) favorise la création d'associations auxquelles il s'adresse ; il ne soutient aucun artisan individuel. Les BA (Bureaux des artisans) ont organisé des groupements d'artisans sur la base de leurs métiers respectifs en vue de la constitution d'une chambre des métiers.

\section{- L'ARTISANAT DU FER, MAILLON ESSENTIEL DANS LE DEVELOPPEMENT DE LA TRACTION ANIMALE}

Les histoires spécifiques de la traction animale au Burkina Faso, au Cameroun et au Sénégal montrent que sa progression et son maintien ne sont possibles qu'avec l'appui des artisans du fer. 


\section{Emprise croissante du secteur artisanal sur le marché des agroéquipements au Nord-Cameroun}

Les artisans du fer assurent 50 p. 100 du marché des agroéquipements (fabrication des charrues, butteurs et charrettes), soit 1700 unités/an, et produisent plus des deux tiers des pièces de rechange. Mais ce ne sont pas forcément les mêmes artisans qui fabriquent les matériels et les pièces. Cinq types d'artisans du fer sont distingués sur la base de leur production qui montre des tendances à la spécialisation (8):

- les forgerons traditionnels, type I (7 p. 100), localisés en zone rurale, ne produisent pas d'outils de traction animale, à l'exception des jougs et des bricoles ;

- les forgeurs de pièces, type II (57 p. 100), se limitent essentiellement à la fabrication des pièces de rechange (socs, contre-seps et versoirs) ;

- les forgeurs d'outils, type III (31 p. 100), produisent davantage des volumes de pièces importants et fabriquent quelques charrues par atelier chaque année

- les menuisiers soudeurs, type IV (2,5 p. 100), ne pratiquent pas la forge, mais dirigent un atelier de "mécanique générale ». Les charrettes représentent une forme de diversification de la production de leurs ateliers (quelques unités par atelier par an);

- les artisans du fer polyvalents, type V (2,5 p. 100), ont une production diversifiée. Ils occupent une place importante du marché artisanal en produisant environ 80 charrues par atelier et par an, et une vingtaine de corps sarcleurs.

Du type I au type IV, les artisans sont de plus en plus jeunes et en majorité lettrés. Leurs ateliers sont plus importants et mieux équipés. Du type II au type V, les chefs d'ateliers ont des stratégies de plus en plus élaborées et la volonté de diversifier leurs produits. Ceci se traduit par des projets d'équipement et par l'amélioration des pratiques d'approvisionnement (stockage du fer et du charbon, collecte systématique du fer dans les villes...), de fabrication (sous-traitance avec des soudeurs ou des forgerons), de gestion (tenue des cahiers de caisse), et de commercialisation (vente aux grossistes, vente hors des limites provinciales).

La vente des pièces et matériels de traction animale produits par les ateliers en activité saisonnière se développe dans deux circuits distincts (8) :

- la vente directe du fabricant à l'utilisateur qui permet l'écoulement de la majeure partie de la production des ateliers ( 83 p. 100 des charrues, 45 p. 100 des corps butteurs, 28 p. 100 des ensembles sarcleurs, 100 p. 100 des charrettes, et 98 p. 100 des pièces de rechange) ;

- un ensemble de points de vente généralement hors des localités de fabrication, mais les revendeurs sont peu expérimentés, peu organisés et ne sont pas formés.

\section{Le secteur artisanal, seul interlocuteur du paysan au Sénégal}

Depuis l'arrêt du PA, les paysans se sont tournés vers les artisans pour la fourniture, la réparation et l'entretien de leurs équipements de traction animale. Mais les forgerons ont des difficultés à réparer et reproduire les pièces usinées du semoir (carter et mécanisme de transmission). Une usure importante de ces pièces rend inutilisable les semoirs. C'est pourquoi, le nombre de semoirs en service diminue, car les achats de semoirs neufs sont faibles, les paysans les trouvant trop chers. Les résultats des études menées sur l'artisanat du fer dans le cadre de l'ATP $(2,7,11)$ confirment ceux des études antérieures de l'Institut sénégalais de recherche agricole (Isra) menées dans les années 1980-90 (4, 12).

Le réseau des artisans du fer est important dans le bassin arachidier. Les artisans ont 40 ans en moyenne et ne sont pas, pour la plupart, inscrits à la chambre des métiers. Ils paient généralement au comptant la matière première, mais ils pratiquent facilement le crédit aux paysans, ce qui leur pose parfois des difficultés de recouvrement. Tous entretiennent des relations professionnelles avec d'autres artisans. Ils ont très souvent des activités secondaires (agriculture, location de matériels) car les travaux sur les matériels agricoles sont saisonniers. On distingue trois grandes catégories d'artisans du fer $(2,3)$, citées ci-après.

Les artisans urbains $(\approx 60$ p. 100) sont basés dans les centres urbains (communes) et parfois dans certaines communautés rurales qui polarisent de grands marchés. Bien équipés (outils manuels et électriques), ils fabriquent une gamme diversifiée d'équipements : outils manuels, pièces d'usure, matériels de traction animale, de battage et d'après-récolte (batteuses à mil, décortiqueuses à mil et à arachide) et de transport. Ils font aussi de la menuiserie métallique et s'approvisionnent chez les ferrailleurs et dans les quincailleries.

Les artisans traditionnels ( $\approx 20$ p. 100) ne disposent pas d'électricité ni de groupe électrogène. Peu équipés et spécialisés dans la forge, ils ne fabriquent que des outils manuels et des pièces d'usure de matériels agricoles. Leur matière première, souvent de médiocre qualité, provient des ferrailleurs. Pour les réparations, leur avantage est d'être proche des paysans.

Les artisans itinérants ( $\approx 20$ p. 100), dont 2 à 3 p. 100 sont équipés de groupe électrogène mobile, commercialisent les pièces fabriquées à l'atelier. Ils réalisent aussi le montage de pièces, la réparation et la vente d'éléments de quincaillerie. Ceux équipés d'un groupe électrogène peuvent effectuer des réparations plus importantes. Ils fréquentent les marchés hebdomadaires jusqu'à quatre fois par semaine, se déplaçant sur des distances importantes par taxi-brousse ou par charrette.

A coté de ces artisans forgerons, les fondeurs fournissent des pièces comme les disques de semoir, les pignons d'entraînement en bronze ou zamak, et les mécaniciens taillent au tour ces mêmes pignons dits adaptables.

La vente des pièces et des matériels d'occasion est très développée et souvent itinérante. Elle est pratiquée sur la majorité des marchés hebdomadaires. Cette activité semble lucrative car certains forgerons itinérants se sont définitivement limités à la commercialisation de pièces et de matériels (2). Les pièces de rechange représentent la majorité des ventes, viennent ensuite les semoirs d'occasion, les houes (Sine, occidentale) et les charrettes. Ces commerçants pratiquent aussi la location de semoirs et de charrettes. Cependant les problèmes de cette filière restent nombreux : équipement des ateliers, disponibilité de matières premières, production de qualité et structuration de la profession.

\section{Les artisans et les PME : un secteur dynamique au Burkina Faso}

Jusqu'en 1998, les PME et les artisans auraient produit environ 50000 charrues, 30000 houes, 15000 butteurs et plus de 100000 charrettes (1). Ces chiffres sont obtenus en retirant des résultats de l'enquête nationale de statistique agricole (Ensa), les matériels produits par le Centre national d'équipement agricole (Cnea) et l'Apicoma, ceux importés du Sénégal, du Mali, de France, mais aussi d'Inde depuis peu, et par la Côte d'Ivoire (matériel italien Otma), et ceux produits par l'Atelier régional de construction de matériel agricole (Arcoma) entre 1957 et 1981.

Pour les PME et les artisans, la production de matériels agricoles est saisonnière, entre mars et juillet pour les matériels aratoires, et septembre et octobre pour les charrettes. C'est pourquoi ces PME et les ateliers artisanaux urbains (soudeurs, menuisiers métalliques, fondeurs, menuisiers bois) diversifient leurs activités. Ainsi, 
ils fabriquent du mobilier, des portes et des fenêtres métalliques. Bien qu'ils possèdent des avantages (disponibilité en énergie électrique, savoir-faire dans la fabrication des matériels agricoles), ces ateliers sont confrontés à des contraintes majeures (éloignement des zones de production agricoles, donc des acheteurs, accès aux crédits et aux financements limités). Ils couvrent les demandes d'ONG et de projets, et celle urbaine en charrettes et autres moyens de transport.

Les PME (sociétés anonymes et privées) sont peu nombreuses et localisées à Ouagadougou et Bobo-Dioulasso. Elles produisent de 1000 à 3000 matériels de traction animale par an, surtout des charrettes pour le marché urbain et, à un moindre degré, des houes Manga et des charrues bovines. La production de matériels agricoles n'est qu'une des activités de ces PME qui fabriquent aussi des portes et des fenêtres et qui, pour certaines, font le commerce des matériaux ferreux et des essieux, mais aussi du transport.

Les ateliers urbains produisent surtout des charrettes, mais très peu de matériels aratoires. Certains artisans forgerons fabriquent aussi les pièces d'usure des matériels agricoles et équipements d'aprèsrécolte pour des ateliers de soudure, les PME et même l'Apicoma. Certains fabriquent, à bas prix avec des matériaux de récupération, à la demande de commerçants des matériels agricoles de médiocre qualité qu'ils revendent sur les marchés.

Les artisans ruraux et forgerons, proches des paysans, réalisent les réparations sur les matériels agricoles et fabriquent des pièces détachées à la demande. Ils ont généralement une activité de production agricole. Ceux équipés de poste de soudure sont sollicités par les paysans et les artisans des villages avoisinants. Quelques-uns parmi ceux formés par le Cnpar fabriquent des matériels aratoires (charrues, houes, corps butteurs) en quantités très limitées : moins de 10 unités par artisan et par an (9). En effet, dans les provinces, l'énergie électrique n'est généralement pas disponible, sauf pour ceux possédant un groupe électrogène, les centres d'approvisionnement en matière d'œuvre sont éloignés et le coût de cette dernière est élevé.

L'Etat est encore présent ponctuellement pour la diffusion de matériels agricoles issus des unités industrielles, voire de l'importation selon les conditions de financement. Des ONG et la Sofitex assurent encore le financement des matériels de traction animale en ayant de plus en plus recours, mais c'est encore timide, à des matériels de fabrication artisanale. Enfin, la vente directe de matériels et de pièces entre les artisans (ou les commerçants) et les paysans se développe.

\section{CONCLUSION}

Dans le contexte de désengagement des Etats, le secteur de construction artisanale et de distribution des équipements agricoles a montré son dynamisme dans des situations où le circuit intégré de fourniture des équipements a brusquement disparu. Ceci révèle des capacités d'adaptation rapides de cette profession. Cependant, sa pérennité ne sera assurée que si elle sait s'adapter à l'évolution du marché des agroéquipements.

\section{Les réseaux d'artisans du fer : des atouts, mais aussi de fortes contraintes}

Dans le domaine de la traction animale, les réseaux d'artisans forgerons sont en mesure de proposer des services de proximité aux paysans et à leurs organisations : réparations et modifications des matériels, fabrication des pièces d'usure et fourniture de boulons et de clés. Ces réseaux sont les mieux placés pour apprécier les besoins des producteurs et répondre à leurs diverses attentes (maintenance, modalités de paiement, offres adaptées aux revenus variés des paysans...).

Mais face à ces atouts, ces réseaux ont des contraintes fortes. La qualité des produits artisanaux est irrégulière et souvent jugée médiocre par les paysans. Ceci est dû à l'utilisation de matière d'œuvre dont la qualité est très variable. Pourtant, les artisans savent reconnaître la qualité d'un acier, mais ils utilisent ce qui est disponible à des prix abordables, car la matière première compte pour plus de la moitié dans les prix de revient des matériels.

L'accès aux crédits d'équipement et à des fonds de roulement pour l'achat de la matière première est difficile pour les artisans, hormis pour les zones couvertes par les programmes d'appui à l'artisanat. Ces programmes déposent des fonds de garantie spécifiques dans des institutions bancaires pour les crédits aux artisans : cas du Percomm et du BA au Burkina Faso...

Le faible niveau d'éducation des artisans est un frein à l'amélioration de la gestion de leurs ateliers. Leur profession n'est pas bien organisée. La saisonnalité de l'activité d'entretien et de fabrication des matériels rend difficile la gestion des ateliers qui doivent satisfaire, en temps et en qualité, une demande tardive des paysans concentrée sur les mois d'avril à juin, pour les matériels de production, et d'août à octobre, pour les équipements de transport et de transformation. Le manque de fonds pour anticiper la fabrication des matériels et constituer des stocks limite fortement le potentiel de production annuelle des artisans et les oblige à diversifier leurs activités : agriculture, constructions métalliques, commerce... La croissance des ateliers, dont les responsables ont acquis des compétences reconnues sur la mécanisation agricole, est limitée par des lacunes dans le fonctionnement et la gestion : élaboration de devis, suivi de la clientèle, organisation du processus de production... Il en résulte des performances économiques très variables et souvent de fausses impressions des artisans sur la rentabilité de leur activité.

\section{Assurer la pérennité du secteur des agroéquipements : un défi}

Le tissu semi-industriel et artisanal de fabrication d'équipements agricoles est récent et fragile dans les pays étudiés. Il reste encore du chemin à parcourir pour former les bases d'une profession reconnue, influente et actrice manifeste du développement agricole de ces pays. Pour cela, il est nécessaire d'accompagner le développement des associations d'artisans et le renforcement du rôle de leurs instances représentatives par des actions d'appui-conseil appropriées. Cela passe aussi par la mise en œuvre de mesures permettant une réduction des prix des matériels et une meilleure compétitivité du secteur artisanal :

- privatisation des dernières entreprises d'Etat et étude des possibilités de diversification de leurs activités ;

- accès des petites entreprises et des artisans aux appels d'offres des programmes d'équipements financés par l'Etat ;

- allègements de la fiscalité sur la matière première et l'importation de matériaux ferreux ;

- amélioration des conditions d'accès à l'énergie ;

- extension des expériences de crédits d'équipements et de fonds de roulement aux artisans fabricant des matériels agricoles.

Parallèlement, il est indispensable de chercher à structurer et formaliser la demande en matériels agricoles de traction animale. Ceci amène plusieurs types de questions : comment améliorer l'approvisionnement en matériels agricoles des paysans ? quel est le rôle des organisations paysannes dont les responsables n'ont aujourd'hui aucune expérience dans ce domaine ? quelle est la demande réelle des paysans ? comment évolue-t-elle? 
1. BORDET D., BONOU A., COULIBALY K., DONESSOUNE R., HAVARD M., OUEDRAOGO S., 1998. Burkina Faso: stratégie de mécanisation agricole. Document I: Rapport résumé et matrice des options. Document II : Rapport principal. Document III : Cartes et tableaux. Document IV : Annexes. Rome, Italie, FAO.

2. DJIBO A., 2001. La filière artisanale de I'agro-équipement, caractérisation et stratégie des acteurs en milieu rural face au désengagement de l'Etat: cas du bassin arachidier (Sénégal). Mémoire de fin d'Etudes, Ensa, Thiés, Sénégal, 81 p.

3. FALL A., N'DAO T., DIAGNE K., M'BODJ M., 2003. Action thématique programmée sur la traction animale au Sénégal : rapport de synthèse. Dakar, Sénegal, Isra, 76 p.

4. HAVARD M., 1987. Le parc de matériels de culture attelée et les possibilités de sa maintenance dans le département de Nioro. Dakar, Sénegal, Isra, 40 p.

5. HAVARD M. 1997. Bilan de la traction animale en Afrique subsaharienne. Perspectives de développement et de recherches. Gembloux, Belgique, faculté des Sciences agronomiques, $73 \mathrm{p}$.

6. HAVARD M., PIROT R., 1997. Les évolutions des marchés des agroéquipements dans les pays francophones d'Afrique subsaharienne. Marchés trop. méditerr., $\mathrm{n}^{\circ} 2690: 1153-1158$.

7. KANTE S., 2003. Les forgerons ruraux au Sénégal : quels services, quelles stratégies d'appui pour la promotion de la traction animale? Dakar, Sénégal, Ancar, 17 p.

\section{Summary}

Pirot R., Havard M., Vall E., Kemtsop G.A., Fall A. Conditions for the Development of Sustainable Draft Animal Power Support Services in Sub-Saharan Africa: Farm Equipment

Starting in the 1960s, programs that aimed to provide draft animal implements to farmers, located in Burkina Faso, NorthCameroon and Senegal, through import, then through local industrial manufacturers, were mainly financed by the output of peanut and cotton crops. In the 1980s and 1990s, drastic reductions in these financing system programs, particularly in Burkina Faso and Senegal, forced national industries to adjust and diversify their production tool. This situation was worsened by the devaluation of the CFA franc currency, which ended up in an increase in equipment prices. To face this brutal evolution, iron craftsmen gradually took over some of the responsibilities for maintenance and repair of draft animal equipment. Then, they started producing more or less complete draft animal equipment, using recovery raw materials to meet financial capabilities of farmers. However the sustainability of the artisanal agricultural equipment sector can only be insured if improvement of the quality of products, management and performances of workshops are guaranteed, as well as the training of craftsman groups to facilitate supply in raw materials and access to markets. In parallel, farmers' organizations must structure and formalize members' needs with regard to agricultural equipment.

Keywords: Animal power - Farm equipment - Maintenance - Repairing - Workshop - Small enterprise - Market Burkina Faso - Cameroon - Senegal.
8. KEMTSOP TCHINDA G.A., ABAKAR O., VALL E., 2002. Les hommes de fer : état du secteur artisanal de I'agro-équipement, atouts, faiblesses et priorités d'intervention. In : Jamin J.Y., Seiny Boukar L. éds, Savanes africaines: des espaces en mutation, des acteurs face à de nouveaux défis. N’Djaména, Tchad, Prasac.

9. LEMPELIUS C., 1991. Le rôle des artisans-forgerons au marché de matériel de la culture attelée du Burkina Faso. Ouagadougou, Burkina Faso, ministère de l'Artisanat, des petites et moyennes Entreprises, 28 p.

10. LE THIEC G., HAVARD M., 1996. Les enjeux du marché des matériels agricoles pour la traction animale en Afrique de I'Ouest. Agric. Dév., $n^{\circ} 11: 39-52$.

11. PIROT R., 2000. Appui méthodologique à la mise en place de I'enquête sur l'étude du secteur des agroéquipements au Sénégal. Montpellier, France, Cirad-CA, 22 p.

12. SOW S.D., 1995. Le parc de matériels de culture attelée et ses contraintes de maintenance dans le bassin arachidier : cas du département de Nioro du Rip. Dakar, Sénegal, Isra, 66 p.

13. VALL E., ABAKAR O., KENIKOU MOUNKAMA C., CUVIER L., KEMTSOP TCHINDA G.A., DONGMO NGOUTSOP A.L., 2000. Contribution de la traction animale à la modernisation du NordCameroun rural de 1950 à 1999 : histoire et actualité. N'Djaména, Tchad, Prasac (premier comité scientifique), 19 p.

14. VANDENDAEL L., 2000. La charrue des paysans du NordCameroun : un outil, son utilisation et sa fabrication. Mémoire de fin d'études, Gembloux, Belgique, FUSAGx, 85 p. + annexes.

\section{Resumen}

Pirot R., Havard M., Vall E., Kemtsop G.A., Fall A. Condiciones de emergencia y de durabilidad de los servicios de apoyo a la tracción animal en Africa sub sahariana: caso de los equipos agrícolas

A partir de los años 1960, en Burkina Faso, al norte de Camerún y de Senegal, los cultivos de cacahuete y de algodón financiaron programas para equipar a los agricultores con material de tracción animal, alimentados por la importación, luego por las fábricas industriales locales. En los años 1980 y 1990, la reducción drástica en los financiamientos de estos programas, principalmente en Burkina Faso y en Senegal, puso en peligro las industrias nacionales, las cuáles se vieron obligadas a reestructurar y diversificar sus herramientas de producción. La situación se agravó con la devaluación del franco Cfa que trajo consigo un aumento en los precios de los materiales. Frente a estos hechos brutales, los artesanos del hierro reaccionaron tomando a su cargo progresivamente el mantenimiento y las reparaciones de los materiales agrícolas de tracción animal. Luego comenzaron a producir con materiales de recuperación de los equipos agrícolas a precios compatibles con los medios financieros de los agricultores. Sin embargo, la durabilidad del sector artesanal de los equipos agrícolas podrá ser asegurada únicamente mediante el mejoramiento de la calidad de los productos, de la gestión y de los rendimientos de los talleres, así como con la formación de grupos de artesanos para facilitar el aprovisionamiento en materias primas y el acceso a los mercados. Paralelamente, las organizaciones de campesinos deben estructurar y formalizar la demanda de equipos agrícolas por parte de los agricultores.

Palabras clave: Energía animal - Equipo de la explotación agraria - Mantenimiento - Reparación - Taller - Empresa pequeña - Mercado - Burkina Faso - Camerún - Senegal. 